\title{
La producción científica reciente en didáctica de la geografía a través de las sociedades geográficas. Declaraciones, publicaciones y proyectos a nivel nacional e internacional
}

\author{
Rafael de Miguel González \\ Universidad de Zaragoza. Facultad de Educación \\ rafaelmg@unizar.es
}

Recibido: mayo de 2017

Aceptado: junio de 2017

\section{Resumen}

En los últimos años, las diferentes asociaciones científicas geográficas han incrementado sus actividades relacionadas con el fomento de la investigación educativa por medio de congresos, proyectos y publicaciones en forma de revistas o de libros. La propia Unión Geográfica Internacional, a través de su Comisión de Educación Geográfica, ha suscrito diversos documentos hasta llegar a la Declaración Internacional sobre Educación Geográfica de 2016. Otras sociedades, como la American Association of Geographers, la European Association of Geographers, la Geographical Association o la Asociación de Geógrafos Españoles, han promovido igualmente el debate y la investigación sobre la didáctica de la geografía en educación primaria y secundaria, debido a los retos que se plantean hoy en día en la enseñanza y el aprendizaje en cuestiones como el currículo, los contenidos en un sistema global y la metodología docente, pero, especialmente, el impacto de las tecnologías de naturaleza geoespacial en el aula.

Palabras clave: didáctica de la geografía; sociedades geográficas; educación geográfica 
Resum. La producció cientifica recent en didàctica de la geografia a través de les societats geogràfiques. Declaracions, publicacions i projectes a nivell nacional $i$ internacional

En els últims anys, les diferents associacions científiques geogràfiques han incrementat les activitats relacionades amb el foment de la investigació educativa per mitjà de congressos, projectes i publicacions en forma de revistes o de llibres. La pròpia Unió Geogràfica Internacional, a través de la seva Comissió d'Educació Geogràfica, ha subscrit diversos documents fins arribar a la Declaració Internacional sobre Educació Geogràfica de 2016. Unes altres societats, com ara l'Associació Americana de Geògrafs, l'Associació Europea de Geògrafs, la Geographical Association o l'Associació de Geògrafs Espanyols, han promogut igualment el debat i la investigació sobre la didàctica de la geografia en educació primària i secundària, a causa dels reptes que es plantegen avui dia en l'ensenyament i l'aprenentatge en qüestions com ara el currículum, els continguts en un sistema global i la metodologia docent, però, especialment, l'impacte de les tecnologies de naturalesa geoespacial a l'aula.

Paraules clau: didàctica de la geografia; societats geogràfiques; educació geogràfica

Résumé. État de la question actualisé en didactique de la géographie: déclarations internationales, publications, projets et sociétés géographiques

Récemment, différentes associations géographiques ont augmenté leurs activités liées à la promotion de la recherche en éducation géographique, à travers des conférences, des projets, des publications comme des revues scientifiques ou des livres. L'Union Géographique Internationale elle-même, par son Comité sur l'éducation géographique, a signé plusieurs documents préalables à la Déclaration Internationale sur l'éducation Géographique de 2016. D'autres sociétés, telles que l'Association Américaine des Géographes, l'Association Européenne des Géographes, la Geographical Association ou l'Association des Géographes Espagnols ont également favorisé la discussion et la recherche sur l'enseignement de la géographie dans l'enseignement primaire et secondaire, en raison des défis dans l'enseignement et l'apprentissage: les programmes scolaires, les contenus géographiques propres au système global, les méthodes d'enseignement, mais surtout l'impact des technologies géospatiales dans la classe.

Mots-clés: enseignement de la géographie; sociétés géographiques; éducation géographique

Abstract. Updating research on geography education: International charters, publications, conferences and geographical societies

In recent years, several scientific geography associations have increased their activities in order to promote educational research through conferences, projects and publications in the form of journals or books. The International Geographical Union, through its Commission on Geographical Education, has signed several documents up to the 2016 International Charter on Geographical Education. Other societies, such as the American Association of Geographers, the European Association of Geographers, the Geographical Association or the Association of Spanish Geographers have also promoted debate and research on the teaching of geography in primary and secondary education. In this sense, the current challenges in teaching and learning include issues related to the curriculum, geography content in a global system and pedagogies, but especially the impact of geospatial technologies in the classroom.

Keywords: geography teaching; geographical societies; geography education 


\section{Sumario}

1. La Unión Geográfica Internacional

2. La Asociación Americana de Geógrafos y el Consejo Nacional de Educación

Geográfica

3. Otras iniciativas internacionales de investigación en educación geográfica
4. La Asociación Europea de Geógrafos

5. Otras iniciativas europeas de investigación en educación geográfica

6. La didáctica de la geografía en España

7. Conclusiones

Referencias bibliográficas

\section{La Unión Geográfica Internacional}

Tras la publicación del Método para la Enseñanza de la Geografía (Unesco, 1965) y del Nuevo Método para la Enseñanza de la Geografía (Graves, 1982), la Comisión de Educación Geográfica (CGE-IGU) preparó la Declaración Internacional sobre Educación Geográfica para su aprobación por parte de la Unión Geográfica Internacional en 1992, en su vigésimo séptimo congreso celebrado en Washington. Este documento abogaba por una educación geográfica universal, enumeraba una serie de contenidos geográficos esenciales para su impartición en los niveles escolares en tanto que «grandes problemas y controversias de nuestra era», planteaba un enfoque social y humanista de la enseñanza del espacio y proponía tres grandes desafíos: la educación internacional, la educación ambiental y la educación para el desarrollo. Para llevar a cabo este triple enfoque, la educación geográfica - a través de sus contenidos temáticos (físicos o humanos) o regionales - precisaría de una serie de requisitos: formación adecuada del profesorado, asignatura independiente con suficiencia horaria, coherencia de la secuencia curricular, recursos didácticos, investigación educativa, cooperación internacional, etc. En fin, la Declaración detallaba todo un cúmulo de principios y de estrategias pedagógicos necesarios para ser aplicados en los sistemas educativos, de manera que no solo se adquirieran conocimientos y habilidades geográficos, sino que también se fomentara la adquisición de unos valores cívicos universales.

Esta Declaración de 1992 fue sucedida por otros dos documentos que se centraban en dos aspectos más concretos de la educación geográfica: la diversidad y la sostenibilidad. La Declaración Internacional sobre la Educación Geográfica para la Diversidad Cultural, de 2000, y la Declaración de Lucerna sobre Educación Geográfica para el Desarrollo Sostenible, de 2007, han servido para contribuir a difundir la Declaración original, pero también para complementar temáticas y enfoques epistemológicos de la propia disciplina científica de la geografía en el ámbito de la educación. Un análisis detallado de la evolución de conceptos, métodos y capacidades educativas que subyacen en estas declaraciones ha sido previamente analizado en De Miguel et al. (2016a).

En los últimos cuatro años, se ha intensificado la acción de la Comisión de Educación Geográfica, con el fin de dar un impulso renovado a estas referen- 
cias internacionales. Así, desde 2013, se ha planteado un texto por año hasta llegar a la revisión de la propia declaración de 1992. Los documentos han sido los siguientes: Declaración conjunta sobre Educación Geográfica en Europa (2013, IV Congreso de EUGEO en Roma), Estrategia Internacional para la Educación Geográfica (2014, Congreso de EUROGEO en Malta y Conferencia Regional de la UGI en Cracovia) y Declaración Internacional sobre Investigación en Educación Geográfica (2015, Conferencia Regional de la UGI en Moscú) (De Miguel y Van der Schee, 2015).

El último Congreso de la UGI en Pekín ha ratificado la nueva Declaración Internacional sobre Investigación en Educación Geográfica (2016) ${ }^{1}$, coincidiendo con la celebración del Año Internacional del Entendimiento Global. La nueva declaración destaca la necesidad de establecer un compromiso de los agentes directos de la educación geográfica. En este sentido, concreta sus anhelos en aspectos como el currículo, el pensamiento crítico, la formación del profesorado, las tecnologías geoespaciales, las metodologías didácticas, la investigación como factor de innovación educativa o la cooperación internacional. Es por ello que la nueva declaración se dirige específicamente a los responsables de política educativa, a los prescriptores del currículo, pero también a los profesores de geografía de todas las naciones del mundo, para ayudar a garantizar que todos los jóvenes reciban una educación geográfica útil, efectiva y de calidad. Para lograrlo, la nueva declaración establece un plan internacional de acción basado en cinco puntos:

1. Lograr que la educación geográfica tenga mayor apoyo social, mayor reconocimiento educativo y más presencia en el currículo.

2. Definir un conjunto de requisitos mínimos para la formación del profesorado que enseñe geografía en educación primaria y secundaria.

3. Reforzar los intercambios de experiencias, metodologías y prácticas pedagógicas significativas.

4. Definir una agenda para la investigación en educación geográfica.

5. Crear una red profesional de profesores de geografía.

Como se comprueba, los congresos de la UGI han sido el ámbito principal de ratificación y difusión de los documentos elaborados por la Comisión de Educación Geográfica. Además, en los congresos de la UGI, existe una clara presencia de la educación geográfica, ya que suele ser una de las líneas temáticas con mayor número de sesiones y aportaciones en ponencias y comunicaciones. Asimismo, en los informes que se presentan allí por parte del Comité Ejecutivo de la UGI, reiteradamente se expresa que la Comisión de Educación Geográfica es una de las más numerosas de toda la UGI en cuanto a miembros, pero también de las más activas debido a la proyección social de la geografía escolar. Sin

1. Su texto completo en español y en inglés ha sido publicado en la revista Didáctica Geográfica, 17 (2016), p. 257-274, mientras que su acceso en línea se realiza a través de la página web de la Comisión (<http://www.igu-cge.org/charters.htm >). 
Tabla 1. Congresos internacionales y conferencias recientes de la Unión Geográfica Internacional

\begin{tabular}{|c|c|c|c|}
\hline Año & Lugar & Carácter & Tema \\
\hline 2017 & La Paz, Bolivia & Conferencia temática UGI & Geographies for peace \\
\hline 2016 & Pekín, China & 33 Congreso UGI & Shaping Our Harmonious Worlds \\
\hline 2015 & Moscú, Rusia & Conferencia regional UGI & $\begin{array}{l}\text { Geography, Culture and Society for } \\
\text { Our Future Earth }\end{array}$ \\
\hline 2014 & Cracovia, Polonia & Conferencia regional UGI & Changes, challenges, responsability \\
\hline 2013 & Kioto, Japón & Conferencia regional UGI & \\
\hline 2012 & Colonia, Alemania & 32 Congreso UGI & Down to earth \\
\hline 2008 & Túnez, Túnez & 31 Congreso UGI & \\
\hline 2004 & Glasgow, Reino Unido & 30 Congreso UGI & One earth, many worlds \\
\hline
\end{tabular}

Fuente: elaboración propia.

embargo, la educación geográfica no ha sido nunca el título de las conferencias recientes, como puede comprobarse en la tabla 1.

La falta de visibilidad de la educación geográfica en los temas principales de los congresos de la UGI no ha sido obstáculo para que este tema sea tratado en sus sesiones específicas, ni para que la propia Comisión de Educación Geográfica lleve a cabo sus propios simposios (tabla 2), en donde se ha podido profundizar, tanto en las declaraciones internacionales como en determinadas líneas específicas de investigación, que en algún caso han dado lugar a publicaciones monográficas.

Algunos de los libros destacados producto del trabajo de la Comisión han sido, entre otros - bien como actas de los simposios, bien como monografías - , los editados por Gerber (2003), por Kent et al. (2004), por Lidstone y Williams (2006), por Purnell et al. (2006), por Reinfried et al. (2007) o por

Tabla 2. Simposios recientes de la Comisión de Educación Geográfica de la Unión Geográfica Internacional

\begin{tabular}{|c|c|c|}
\hline Año & Lugar & Tema \\
\hline 2017 & Lisboa, Portugal & $\begin{array}{l}\text { Integrating Knowledge and Understanding in Geography Educa- } \\
\text { tion }\end{array}$ \\
\hline 2016 & Singapur & Geography that matters. Empowering learners for a diverse world \\
\hline 2015 & Londres, Reino Unido & The power of geographical thinking \\
\hline 2012 & Friburgo, Alemania & Experience-based Geography Learning \\
\hline 2011 & Londres, Reino Unido & Curriculum making in geography \\
\hline 2010 & Estambul, Turquía & $\begin{array}{l}\text { Representations of cultural differences through geographical } \\
\text { education }\end{array}$ \\
\hline 2008 & Túnez, Túnez & \\
\hline 2007 & Lucerna, Suiza & Teaching Geography for Sustainable Development \\
\hline 2006 & Brisbane, Australia & Geographical Education in a Changing World \\
\hline 2004 & Glasgow, Reino Unido & \\
\hline
\end{tabular}

Fuente: elaboración propia. 
Whewell et al. (2011). Junto a las publicaciones en formato de libro, la Comisión de Educación Geográfica impulsó la edición de la revista International Research in Geographical and Environmental Education, incluida en la base de datos SCOPUS. A pesar de las dificultades iniciales, de su desarrollo posterior o de los cambios recientes, los presidentes anteriores de la Comisión realizan un balance en el momento en que dejan la coedición de la revista (Stoltman y Lidstone, 2017).

Merece la pena incluir en este apartado una última iniciativa de la Comisión de Educación Geográfica: la serie editorial, publicada por Springer, «International Perspectives on Geographical Education», que lleva ya dos números editados, uno sobre el proyecto Geoprogressions luego citado (Muñiz et al., 2016) y otro como resultado del Simposio de Londres 2015 sobre el conocimiento geográfico (Brooks et al., 2017); mientras que un tercer libro, sobre la comprensión global, también verá la luz este año (Demirci et al., 2017).

\section{La Asociación Americana de Geógrafos y el Consejo Nacional de Educación Geográfica}

La Asociación Americana de Geógrafos (AAG) celebra anualmente su congreso anual, que se convierte, por derecho propio, en el principal evento científico del mundo en cuanto a participación, por delante incluso de las mismas conferencias de la UGI, aunque sin tanta diversidad de procedencias como estas. En el último Congreso de 2017, celebrado en Boston, se alcanzó la cifra de 6.900 intervenciones entre ponencias, comunicaciones, talleres, pósteres, mesas redondas, etc. De manera proporcional, es en este congreso en el que se expone un mayor número de investigaciones científicas relacionadas con la didáctica de la geografía. En el citado de 2017, 191 presentaciones o pósteres incluyeron el término education como palabra clave de su investigación.

La Asociación Americana de Geógrafos desarrolla igualmente una serie de proyectos educativos, bien en coalición con el Centro Nacional de Investigación en Educación Geográfica (NCRGE, Texas University of San Marcos), como GeoProgressions o GeoSTEM, Geocapabilities (De Miguel, 2016), bien con otros socios, como Esri en GeoMentors, como la NASA en GeoStart, etc.

De entre las publicaciones de la Asociación, destaca Annals of the Association of American Geographers, editada desde 1911, situada en octavo lugar del JCR y que incluye varios artículos sobre educación geográfica.

Por su parte, el Consejo Nacional de Educación Geográfica (NCGE) constituye la principal asociación científica exclusivamente compuesta por geography educators en el sentido amplio del término, tanto profesores norteamericanos de geografía en el sistema escolar K-12 como docentes de la geografía y universitarios encargados de la formación del profesorado, además de miembros de varias ONG, fundaciones, empresas y administraciones educativas estadounidenses relacionadas con la enseñanza de la geografía. Su congreso anual, inferior en número de participantes al de la AAG, es, no obstante, el evento científico que mayor número de investigadores en didáctica de la geografía concentra, debido a su especialización. 
Esa misma razón es la que ha propiciado que el NCGE edite la única revista científica que se dedica monográficamente a la educación geográfica, incluida en la base de datos JCR, Journal of Geography. Uno de los artículos de referencia de esta revista (Baker et al., 2015) establece una agenda para la investigación en las relaciones entre educación geográfica y tecnología geoespacial, exponiendo la carencia de una acumulación de conocimiento científico mejor estructurado, más sistemático y más reproducible, y diseñando cuatro ejes de investigación didáctica: la relación entre las tecnologías de información geográfica y el pensamiento espacial; las dificultades de aprendizaje geográfico con tecnología geospacial; el diseño curricular a partir de tecnología geospacial, y el desarrollo profesional de los docentes. Adicionalmente, el NCGE publica una segunda revista, de menos impacto científico pero de mayor impacto escolar y difusión entre los docentes en geografía, por recoger experiencias de aula, proyectos escolares y reflexiones sobre actividades innovadoras.

Sin embargo, las dos aportaciones adicionales, de extraordinaria importancia para la didáctica de la geografía, realizadas por estas dos instituciones norteamericanas citadas, se han producido cuando han actuado cooperando entre sí, pero también con la National Geographic y con la American Geographical Society. Se trata de los proyectos Geography for Life (Heffron y Downs, 2012) y Road Map for 21st Century Geography Education (Bednarz et al., 2013). El primero de ellos ha supuesto la definición del cuarto documento - desde 1963 - de estándares nacionales para el aprendizaje de la geografía en la educación primaria y secundaria, introduciendo la cuestión del pensamiento espacial y su vinculación con las tecnologías geoespaciales en el currículo escolar (National Research Council, 2006). El segundo proyecto se ha sustanciado en un documento a modo de libro blanco de la educación geográfica americana, dividido en tres partes, realizadas respectivamente por tres comités, cada uno de los cuales ha redactado un informe particular sobre la temática trabajada: materiales didácticos y desarrollo profesional; evaluación en educación geográfica, e investigación en educación geográfica.

\section{Otras iniciativas internacionales de investigación en educación geográfica}

Continuando con el ámbito anglosajón, existen otras publicaciones que han recogido estudios comparativos, a escala internacional, sobre diferentes temas de análisis relacionados con la didáctica de la geografía. Además de algún trabajo pionero (Stoltman, 1976; Marsden, 1984; Hill, 1992; Haubrich, 1982; Gerber y Williams, 2002), donde se constata la presencia y el impulso de presidentes anteriores de la Comisión de Educación Geográfica de la UGI, la citada editorial Springer ha publicado dos monografías relacionadas con la creciente importancia de las tecnologías de información geográfica en educación (Milson et al., 2012; Muñiz et al., 2015). Estos libros, junto con los anteriormente citados de la misma editorial impulsados por la Comisión de Educación Geográfica, permiten publicar capítulos y mostrar aportaciones 
de comunidades de enseñantes de la geografía, menos conocidas por su menor proyección exterior, pero no por ello menos relevantes debido a los países $-\mathrm{y}$ a los correspondientes sistemas educativos - que representan: Rusia, China, India, Sudáfrica, Canadá, Japón, etc.

En fin, la lista de publicaciones de referencia internacional sobre didáctica de la geografía editadas a partir del año 2000 ha sido muy relevante y enriquecedora. Puede consultarse la selección que se muestra en la página web de la Comisión. De todas ellas, han tenido un impacto especial en la comunidad internacional de geógrafos especializados en educación -la mayor parte por su tratamiento general de la clase de geografía a modo de manuales de didáctica de la geografía para los niveles de educación primaria y secundaria - las escritas por Gersmehl (2008), Catling y Willy (2009), Lambert y Morgan (2010), Standish (2012), Lambert y Jones (2013), Butt (2015) o Kerski (2016).

Pasando de los libros a las revistas científicas, la segunda de ellas dedicada monográficamente a la enseñanza de la geografía, e incluida en la base de datos JCR, es Journal of Geography in Higher Education. A pesar de que su temática principal es la enseñanza de la geografía en niveles universitarios, su interés por la didáctica de la geografía en educación escolar demuestra el habitual error en denominarla no universitaria. La razón principal es que la formación del profesorado de geografía, tanto en primaria como en secundaria, es de nivel universitario. En los niveles de grado y de máster, en las universidades de todo el mundo, no solo se enseña geografía como disciplina científica a nivel especulativo, como preparación para salidas profesionales relacionadas con el urbanismo, el medio ambiente o el paisaje, o como técnico en sistemas de información geográfica y teledetección, sino que también se enseña geografía para ser profesor de geografía, tal y como expusimos en esta publicación para el caso español (De Miguel y De Lázaro (2016), utilizando las mismas estrategias de aprendizaje en educación secundaria que en educación superior; por ejemplo: la tecnología geoespacial, las salidas de campo, el aprendizaje basado en problemas territoriales, por mencionar tres muestras de temáticas reiteradas constantemente en los artículos publicados en esta revista. Un dato adicional permite entender esta solución de continuidad en la preocupación común por la enseñanza y el aprendizaje de la geografía en niveles escolares y universitarios. La edición de esta revista ha corrido a cargo durante mucho tiempo de dos expresidentes de las dos asociaciones antes citadas: Robert Bednarz, que lo fue de la NCGE, y Sarah W. Bednarz, que lo fue de la AAG. Otras revistas de geografía de impacto, como GeoJournal o International Journal of Digital Earth, han publicado artículos sobre educación geográfica, pero de manera dispersa e irregular, a pesar de que algunos trabajos han alcanzado una gran difusión (Bednarz, 2004; Kerski, 2008).

Por concluir con el ámbito anglosajón, es destacable el esfuerzo realizado recientemente por el equipo de educación de ESRI y la amplitud de recursos disponibles en <http://edcommunity.esri.com>, para la enseñanza de la geografía y para la investigación en didáctica de la geografía, especialmente tras el 
lanzamiento del sistema de información geográfica en la web ArcGIS Online, que permite desarrollar el pensamiento espacial, pero también el aprendizaje geográfico por descubrimiento o basado en la indagación (Roberts, 2013), tal y como hemos tenido la oportunidad de desarrollar con el Atlas Digital Escolar (De Miguel et al., 2016b).

Dos ámbitos geográficos menos próximos y conocidos han desarrollado una estructura organizativa e investigadora en didáctica de la geografía. La Southeast Asian Geography Association (SEAGA) lleva, desde 1990, organizando congresos bianuales de educación geográfica, cuyas actas ha ido editando de manera consecutiva, recogiendo aportaciones de geógrafos del sudeste asiático. Por su parte, la Australian Geography Teachers Association (AGTA) también celebra eventos científicos cada dos años. Además de difundir recursos didácticos, boletines de noticias, proyectos curriculares, etc., edita la revista Geographical Education.

Por último, pero no por ello menos importante, la comunidad iberoamericana ha conocido igualmente un notable desarrollo investigador en didáctica de la geografía, a pesar del desigual reconocimiento de esta disciplina en los respectivos currículos escolares nacionales (De Miguel et al., 2016a). En 2007, durante el XI Encuentro de Geógrafos de América Latina en Bogotá, se decidió crear la Red Latinoamericana de Investigadores en Didáctica de la Geografía, denominada Redladgeo. En su página web (<www.geopaideia.com $>$ ), puede constatarse el balance de la interesante producción científica llevada a cabo, que hemos tenido en ocasiones la oportunidad de conocer de primera mano cuando sus investigadores han visitado España y han participado en nuestros congresos de didáctica de la AGE. La red Redladgeo ha celebrado tres coloquios internacionales (Sao Paulo en 2010, Santiago de Chile en 2012 y Buenos Aires en 2014), ha desarrollado varios proyectos de investigación, ha coordinado diversos libros (Castellar et al., 2012) y ha editado la revista Anekumene, cinco números entre 2011 y 2013.

Una segunda iniciativa, complementaria de la anterior, es GeoForo, el Foro Iberoamericano sobre Educación, Geografía y Sociedad (<www.geoforo.com>). Aunque impulsado inicialmente desde España e inserto en el portal Geocrítica (Souto y Fita, 2014), tiene una gran difusión sobre los investigadores en didáctica de la geografía en países como Portugal, Colombia, Brasil, Argentina, Venezuela o Chile. Tiene publicado veintitrés foros en la red, con la activa participación de geógrafos de esos países, además de un foro extraordinario sobre construcción de unidades didácticas.

Cada uno de estos países tiene una expresión dispar en la producción investigadora, bien sea en Chile (Conway y Araya, 2011); en Brasil, con las revistas Giramundo o Revista Brasileira de Educação Geográfica; en Argentina, con la Red de Docentes e Investigadores en la Enseñanza de la Geografía en las universidades argentinas (Redieg) (Lorda y Prieto, 2016), con el portal Geoperspectivas o con los Encuentros Nacionales de Profesores de Geografía (23 ediciones hasta el momento), o, en Colombia, con el grupo de investigación Geopaideia. 


\section{La Asociación Europea de Geógrafos}

En 1979 se constituyó la European Standing Conference of Geography Teachers' Associations (ESCGTA), a modo de red de asociaciones de geógrafos en los países de la entonces Comunidad Económica Europea. Sus dos actividades principales eran la celebración de una conferencia bianual en Bruselas, desde 1980, y la publicación de la revista EUROGEO: Geographical Bulletin, desde 1981. A partir del año 1994, la asociación se amplió para albergar no solo a los países de la UE, sino también a los de toda Europa. Fue entonces cuando tomó el nombre de EUROGEO, cuyos estatutos ha modificado posteriormente para abrirse a todos los países del mundo, y no únicamente a asociaciones de geógrafos, sino también a geógrafos como miembros individuales. Este hecho, unido a la obtención de proyectos europeos coordinados por la Universidad de Liverpool, supuso el relanzamiento de la asociación. El primer proyecto obtenido en 1999 fue EUROGEO (programa Minerva), que estaba centrado en promocionar la ciudadanía y la dimensión europea a través de la enseñanza de la geografía, creando materiales y recursos en la incipiente red digital.

Este proyecto concluyó con la Conferencia de 2001 en Liverpool, en donde se decidió solicitar otro proyecto Socrates-Erasmus, cuya concesión fue el relanzamiento definitivo de EUROGEO: HERODOT: Network for Geography in Higher Education, que tuvo dos fases (2002-2005 y 2006-2009). Además, en la conferencia siguiente de 2002, en Funchal, el profesor de la Universidad Hope de Liverpool, Karl Donert, accedió a la presidencia de EUROGEO, cargo que ha mantenido hasta la actualidad, lo cual ha permitido una estabilidad, un crecimiento y un reconocimiento internacional de esta asociación, pero también de la didáctica de la geografía en el ámbito científico de nuestra disciplina.

La red HERODOT estuvo dedicada al impulso de la geografía en la enseñanza superior, en el marco del proceso de Bolonia, organizando múltiples eventos científicos organizados por EUROGEO, lo que favoreció el trabajo en red entre diversas asociaciones e instituciones europeas, fomentando foros de intercambio de experiencias y difundiendo buenas practicas docentes, profesionales e investigadoras en Europa, no solo entre docentes e investigadores, sino también entre los jóvenes geógrafos. Al final de su existencia, la red alcanzó más de 220 miembros, incluyendo una gran parte de departamentos universitarios de geografía de Europa, pero también de otras zonas del mundo.

La financiación del proyecto permitió pasar de las conferencias bianuales a las conferencias anuales, y llevarlas a diferentes ciudades europeas, involucrando así a las comunidades locales y nacionales de geógrafos. Hasta la fecha actual, las científicas llevadas a cabo por EUROGEO (ESCGTA, hasta 1994) han sido las que se detallan en el cuadro 3.

Una vez concluido el proyecto HERODOT, EUROGEO lideró otro diferente, que contaba con la financiación del programa "Comenius», denominado Digital-earth.eu: Geomedia in schools, del que han formado parte más de cincuenta instituciones europeas, tanto universitarias como centros de educación secundaria. El objetivo del proyecto es impulsar la utilización de los medios 
Tabla 3. Conferencias de EUROGEO

\begin{tabular}{|c|c|c|c|}
\hline Año & Lugar & $\begin{array}{l}\text { Institución } \\
\text { coorganizadora }\end{array}$ & Tema \\
\hline 1980 & Bruselas, Bélgica & & \\
\hline 1982 & Bruselas, Bélgica & & \\
\hline 1984 & París, Francia & & \\
\hline 1986 & Bruselas, Bélgica & & \\
\hline 1988 & Bruselas, Bélgica & & \\
\hline 1990 & Bruselas, Bélgica & & \\
\hline 1992 & Bruselas, Bélgica & & \\
\hline 1994 & Bruselas, Bélgica & & \\
\hline 1996 & Salzburgo, Austria & & \\
\hline 1998 & Luxemburgo & $\begin{array}{l}\text { Luxembourg Geography } \\
\text { Association }\end{array}$ & Geography in Europe \\
\hline 2001 & Liverpool, Reino Unido & Universidad de Hope & $\begin{array}{l}\text { Teaching geography, skills and } \\
\text { curricula }\end{array}$ \\
\hline 2002 & Funchal, Portugal & $\begin{array}{l}\text { APG, Associação de } \\
\text { Professores de Geografia }\end{array}$ & $\begin{array}{l}\text { Geography teaching in an enlarging } \\
\text { Europe }\end{array}$ \\
\hline 2004 & Bled, Eslovenia & $\begin{array}{l}\text { University of Ljubljana, } \\
\text { Slovenian Geography } \\
\text { Teacher Association }\end{array}$ & New developments in Geography \\
\hline 2005 & Torún, Polonia & $\begin{array}{l}\text { Institute of Geography, } \\
\text { Nicolaus Copernicus } \\
\text { University }\end{array}$ & $\begin{array}{l}\text { Changing Horizons in Geography } \\
\text { Education }\end{array}$ \\
\hline 2007 & Estocolmo, Suecia & Stockolm University & $\begin{array}{l}\text { Geography for Society: Putting } \\
\text { Bologna into Action }\end{array}$ \\
\hline 2008 & Liverpool, Reino Unido & Liverpool Hope University & Future Prospects in Geography \\
\hline 2009 & Ayvalik, Balikesir, Turquía & Balikesir University & Celebrating Geographical Diversity \\
\hline 2010 & Praga, República Checa & Charles University & Sustainable Geographies \\
\hline 2011 & Atenas, Grecia & $\begin{array}{l}\text { Laboratory of Geography } \\
\text { of the National Technical } \\
\text { University of Athens }\end{array}$ & $\begin{array}{l}\text { Geography: Your world- } \\
\text { A European Perspective }\end{array}$ \\
\hline 2012 & Dublín, Irlanda & $\begin{array}{l}\text { St. Patrick's University } \\
\text { College }\end{array}$ & $\begin{array}{l}\text { Geography and Global Understan- } \\
\text { ding: Connecting the Sciences }\end{array}$ \\
\hline 2013 & Brujas, Bélgica & Universidad de Gante & $\begin{array}{l}\text { Geography: Linking Tradition and } \\
\text { Future }\end{array}$ \\
\hline 2014 & La Valleta, Malta & Universidad de Malta & $\begin{array}{l}\text { The Power of Geography and the } \\
\text { Role of Spatial Information }\end{array}$ \\
\hline 2015 & Ankara, Turquía & $\begin{array}{l}\text { Turkish Association } \\
\text { of Geographers, Gazi } \\
\text { University }\end{array}$ & $\begin{array}{l}\text { Communicating Geography: } \\
\text { Serving our world }\end{array}$ \\
\hline 2016 & Málaga, España & $\begin{array}{l}\text { Universidad de Málaga, } \\
\text { AGE, Real Sociedad } \\
\text { Geográfica }\end{array}$ & $\begin{array}{l}\text { Geographic Information: } \\
\text { For a better world }\end{array}$ \\
\hline 2017 & Ámsterdam, Holanda & Universidad de Utrecht & $\begin{array}{l}\text { Challenges for geographical } \\
\text { education }\end{array}$ \\
\hline
\end{tabular}

Fuente: actualizado de De Lázaro (2013). 
tecnológicos digitales en el aprendizaje escolar de la geografía, lo que se ha denominado geo-media, debido a la reciente irrupción de globos virtuales, geovisores, SIGWeb, teledetección y aplicaciones en dispositivos móviles. Toda la diversidad de cartografía digital, en sus múltiples soportes y formatos, está revolucionando la manera de enseñar y de aprender geografía en la escuela, de manera que el proyecto Digital-earth.eu ha supuesto un avance en la reflexión didáctica en cuatro aspectos fundamentales: los recursos didácticos digitales; las metodologías de enseñanza y aprendizaje; la formación del profesorado, y el currículo escolar. En fin, otros proyectos internacionales han permitido que la asociación desarrollara líneas complementarias de investigación relacionadas con la didáctica de la geografía: iGuess, Piri Reis, Spacit, i-Use, Geocapabilities, Geoskills Plus, School on the cloud, GI-Learner, YouthMetre.

La evidencia de producción científica de EUROGEO se ha plasmado además en sus publicaciones. Por una parte, las revistas científicas. La revista inicial que, de hecho, acabó dando nombre a la asociación, EUROGEO, Geographical Bulletin, dejó de publicarse a finales de los años noventa. Sin embargo, en 2010, se lanzó European Journal of Geography como publicación que recogiera las aportaciones a las conferencias, de los proyectos citados o de investigaciones de sus socios. Con cuatro números anuales, actualmente está incluida en la base de datos SCOPUS. Por otra parte, los dos principales proyectos han permitido editar diversos libros, tanto de HERODOT (Schmeinck, 2009; Donert, 2010) como de Digital-earth.eu (Jekel et al., 2012; De Miguel y Donert, 2014).

Además de todo esto, EUROGEO colabora activamente con las otras entidades dedicadas a la promoción de la geografía y de la educación geográfica (Higgitt et al., 2008), como las citadas Unión Geográfica Internacional y Asociación Americana de Geógrafos, pero también de EGEA, European Association of Young Geographers. Igualmente, su labor en la promoción de la geografía como disciplina social le ha permitido un reconocimiento, tanto en la Conferencia Internacional de ONG del Consejo de Europa - y en el Comité de Democracia, Cohesión Social y Retos Globales-, como en el Comité de ONG del Consejo Económico y Social de las Naciones Unidas, en donde tiene igualmente participación en agencias como UNEP o en la General Assembly of Partners, de UNHABITAT.

\section{Otras iniciativas europeas de investigación en educación geográfica}

La segunda asociación geográfica europea es EUGEO, que funciona como una federación de las diferentes asociaciones nacionales de geógrafos existentes en nuestro continente. Debido a este carácter, no tiene una actividad tan propia como EUROGEO en materia de publicaciones y proyectos, pero sí que organiza congresos anuales, en los que la didáctica de la geografía no está tan presente, por no ser siempre una prioridad en las respectivas sociedades geográficas nacionales.

Por el contrario, de manera adicional a la revista previamente citada, hay dos revistas específicas de alcance europeo que merecen ser reseñadas: J-Rea- 
ding: Journal of Research and Didactics in Geography es una iniciativa de la Asociación Italiana de Profesores de Geografía (AIIG), pero que cuenta con el respaldo de EUGEO. Desde que inició su andadura en 2012, publica dos números anuales.

Desde una iniciativa particular surgida en Turquía, con apoyo inicial de la Geographical Association, en 2011, se comienza a editar Review of International Geographical Education Online (RIGEO), con tres números anuales, en donde se han publicado, entre otros, destacados trabajos referidos a la metodología de investigación en didáctica geográfica (Zadrozny et al., 2016).

El Reino Unido dispone de la estructura más potente a nivel nacional en lo referente a la didáctica de la geografía. La Royal Geographical Society (with Institute of British Geographers) dedica una parte relevante de sus actividades a la promoción y a la investigación de la educación geográfica, además de organizar la certificación oficial para la docencia, en la figura del Chartered Geographer (Teacher). Como la propia web de la sociedad indica, su «Departamento de Educación está en el corazón del trabajo de la sociedad», lo que se constata en la enorme oferta de recursos didácticos, cursos o proyectos, como el «Global learning programe».

Además, el Reino Unido dispone de una entidad independiente de alrededor de 5.000 profesores de geografía asociados: la Geographical Association. Constituye, junto con la norteamericana NCGE, la más potente organización especializada en didáctica de la geografía, estrechamente orientada a la formación inicial y continua del profesorado de dicha materia por medio de la innovación y la investigación didáctica. Su labor en los procesos de revisión del National Curriculum, de la reforma de las pruebas externas (GCSE, A levels), su ingente banco de manuales escolares, cartografía, materiales curriculares y recursos didácticos, sus proyectos educativos (por ejemplo: el citado Geocapabilities, en consorcio con EUROGEO y AAG, entre otros), su amplia oferta de cursos y su conferencia anual (celebrada casi siempre entre Manchester, Derby y Guilford) permiten que el profesor de geografía británico tenga una referencia y un soporte profesional de incalculable valor, que se incrementa con las tres revistas que edita: Primary Geography, Teaching Geography y Geography, an International Journal.

En Francia, a pesar de que la pionera Societé de Geographie se crea en París en 1821, no existe una organización tan evidente de los profesores de geografía. Entre otras razones, ello es debido a que dicha materia se enseña junto a la historia en los tres niveles de école-collège-lycée (Mével y Tutiaux-Guillon, 2013). La revista Historiens et Géographes, de la Asociación de Profesores de Geografía e Historia, apenas edita artículos de geografía. En esta publicación, está mucho más presente la historia. El Comité National Français de Géographie no dispone de una comisión específica de didáctica de la geografía, sino que los temas educativos se integran en otra más general: la comisión de epistemología, historia y enseñanza de la geografía. A pesar de todos estos condicionantes, un grupo de pedagogos de la geografía ha creado el portal Didageo, en donde difunden eventos científicos, publicaciones (Robic y Rosemberg, 2016), 
recursos y resultados de investigación, en buena parte relacionados con la aplicación de las tecnologías de la información geográfica en el aula. Y, al margen de todas estas circunstancias, en Francia se desarrolla, desde el año 1990, un acontecimiento anual relacionado con la enseñanza de la geografía: el Festival Internacional de Geografía, en Saint-Dié-des-Vosges, en donde precisamente se concede el mayor galardón científico en dicho ámbito, el premio Vautrin-Lud.

En Alemania, la Sociedad Alemana de Geografía, o Deutsche Gesellschaft für Geographie (DGfG), agrupa, entre otras, a la Hochschulverband für Geographie und ihre Didaktik (HGD) y a la Verband Deutscher Schulgeographen (VDSG). La primera es una asociación universitaria de profesores de Didáctica de la Geografía, mientras que la segunda es una asociación de profesores de geografía en los centros escolares (Kulke et al., 2004). Esta acción consorciada ha permitido trabajar en un documento para la definición del currículo en el ámbito federal, que constituye un referente en la didáctica de la geografía alemana: Educational Standards in Geography for the Intermediate School Certificate with sample assignments (2012, ed. en inglés).

\section{La didáctica de la geografía en España}

La didáctica de la geografía en España es una disciplina relativamente reciente, debido a su adscripción al área de conocimiento de la Didáctica de las Ciencias Sociales, tras su creación por el conocido Real Decreto de finales de 1984, en desarrollo de la Ley de reforma universitaria. Además, la docencia que se ha llevado a cabo durante más de dos décadas ha correspondido exclusivamente a la educación infantil y primaria, sin perjuicio del extinto Certificado de Aptitud Pedagógica. Dicho en otras palabras, hasta la implantación del máster de Profesorado en Educación Secundaria, en el curso 2009-2010, no ha existido una docencia universitaria plena en didáctica de las ciencias sociales y, en consecuencia, en didáctica de la geografía, en el sentido de abarcar la formación pedagógica del profesorado para el conjunto de niveles educativos, máxime cuando, desde la implantación de la LOGSE, la geografía como asignatura autónoma solo se imparte en el curso de segundo de bachillerato.

Ello no ha sido obstáculo, por otra parte, para que se fuera pergeñando una incipiente línea de investigación en didáctica de la geografía desde diferentes ámbitos: los trabajos pioneros de Vilá-Valentí en el seno de la Comisión de Educación Geográfica de la UGI durante los años sesenta y setenta (De Miguel et al., 2016a), las publicaciones de Geocritica: Cuadernos Críticos de Geografía Humana (Bailey, 1981; Capel et al., 1984), los trabajos de profesores universitarios o de bachillerato (el propio Horacio Capel, Agustín Hernando, María Jesús Marrón, Adela Gil Crespo y Xosé Manuel Souto), las tesis doctorales de los citados Agustín Hernando en 1979 y Alberto Luis en 1984, los artículos verdaderamente de didáctica —y no de epistemología — en los primeros números de la primera época de la revista Didáctica Geográfica, sobre todo diversas aportaciones de Pedro Plans, José Luis Andrés, José Luis González Ortiz, etc. Como puede comprobarse, tanto en las actas de las Primeras Jor- 
Tabla 4. Congresos del Grupo de Didáctica de la Geografía, de la Asociación de Geógrafos Españoles.

\begin{tabular}{llll}
\hline Año & Lugar & Universidad & Alcance \\
\hline 1988 & Madrid & UAM & Primeras Jornadas de Didáctica de la Geografía \\
1991 & Burgos & UBU & Segundas Jornadas de Didáctica de la Geografía \\
1996 & Madrid & UCM & Terceras Jornadas de Didáctica de la Geografía \\
1998 & Alicante & UAL & IV Congreso Nacional de Didáctica de la Geografía \\
2000 & Murcia & UMU & V Congreso Nacional de Didáctica de la Geografía \\
2001 & Madrid & UCM & I Congreso lbérico de Didáctica de la Geografía \\
2003 & Toledo & UCLM & VI Congreso Nacional de Didáctica de la Geografía \\
2005 & Lisboa & UDL & II Congreso Ibérico de Didáctica de la Geografía \\
2006 & Almagro & UCLM & III Congreso lbérico de Didáctica de la Geografía \\
2007 & Valencia & UDV & VII Congreso Nacional de Didáctica de la Geografía \\
2008 & Jaén & UDJ & VIII Congreso Nacional de Didáctica de la Geografía \\
2009 & Lisboa & UDL & IV Congreso lbérico de Didáctica de la Geografía \\
2010 & Madrid & UCM & IX Congreso Nacional de Didáctica de la Geografía \\
2011 & Málaga & UMA & V Congreso Ibérico de Didáctica de la Geografía \\
2012 & Zaragoza & UZA & I Congreso Europeo de Didáctica de la Geografía \\
2013 & Oporto & UDO & VI Congreso Ibérico de Didáctica de la Geografía \\
2014 & Córdoba & UCO & X Congreso Nacional de Didáctica de la Geografía \\
2015 & Alicante & UAL & VII Congreso Ibérico de Didáctica de la Geografía \\
2016 & Sevilla & UPO & XI Congreso Nacional de Didáctica de la Geografía \\
2017 & Lisboa & UNL & VIII Congreso lbérico de Didáctica de la Geografía \\
\hline
\end{tabular}

Fuente: actualizado de Jerez y Córdoba (2015).

nadas de Didáctica de la Geografía como en un trabajo previo (Tovar et al., 1980), como también en las referencias de los dos citados cuadernos didácticos de Geocrítica, la bibliografía española en didáctica de la geografía era realmente escasa hasta mediados de la década de 1980.

A esta situación científica de debilidad investigadora en didáctica de la geografía, le pone remedio la creación del Grupo de Didáctica de la Geografía, de la Asociación de Geógrafos Españoles en $1986^{2}$, con la puesta en marcha de las jornadas de didáctica, luego congresos nacionales (e ibéricos, e incluso europeo), que han sido la principal referencia para cualquier investigación en didáctica de la geografía hasta la fecha actual. Bien puede decirse que toda persona que ha desarrollado cualquier tipo de producción científica en didáctica de la geografía en España ha participado en los congresos del grupo. Esa consolidación de dicha disciplina en nuestro país se ha visto expresada de diferentes maneras, pero siempre ha tenido un denominador común: su vinculación en mayor o menor grado con el grupo. El Grupo de Didáctica de la AGE y sus veinte congresos propios celebrados desde entonces (tabla 3)

2. En la presentación de su propia web (<http://www.age-geografia.es/didacticageografia/index. $\mathrm{php}$ ? $\mathrm{m}=1>)$, se encuentra un resumen de la historia del grupo. 
han permitido la promoción profesional de profesores universitarios - pero también de profesores de geografía en secundaria y primaria entregados a la innovación y a la investigación-, han constituido el marco conceptual de las tesis doctorales realizadas desde entonces, han permitido la difusión de proyectos de investigación y de recursos didácticos (entre ellos, los derivados del convenio con el IGN, España a través de los mapas y Paisajes españoles), han fomentado la participación del curso de verano en la Universidad Carlos III, etc. En definitiva, han dado solidez y estabilidad a la didáctica de la geografía, en niveles casi similares a los que hemos visto en el ámbito anglosajón.

Las actas de todos estos congresos, disponibles en la página web del grupo, constituyen, sin duda alguna, el principal corpus científico de la didáctica de la geografía en España, junto con los manuales de referencia (Marrón y Moreno, 1995; Souto, 1998; Comes, 1998; Sebastiá y Tonda, 2017) y la revista Didáctica Geográfica. Gracias al impulso de la presidenta del Grupo que más tiempo ha ejercido en ese puesto, María Jesús Marrón, se decidió recuperar, en 1996, la revista Didáctica Geográfica en su segunda época, en este caso, dedicada monográficamente a su título. En este segundo periodo, se han publicado dieciocho números, con periodicidad prácticamente anual, por el esfuerzo decidido de la entonces presidenta del Grupo, pero también de sus sucesoras, María Luisa de Lázaro y Emilia Tonda. El balance de esta ingente producción científica, actas de congresos y revista, ya se ha realizado y a ella nos remitimos (Sebastiá y Tonda, 2011; Sebastiá y Tonda, 2012; Tonda y Sebastiá, 2012; De Lázaro et al., 2013; Sebastiá y Tonda, 2015; Jerez y Córdoba, 2015).

La segunda asociación, primera en el tiempo por su fundación en 1876, es la Real Sociedad Geográfica. Su vinculación con la didáctica de la geografía es menor, pero no por ello menos importante, debido a su proyección internacional (De Lázaro et al., 2014), por haber sido acreditada como centro de excelencia Digital-earth.eu, ya que es una de las entidades fundadoras de EUROGEO, así como por formar parte del Secretariado del Comité Español UGI (junto a la AGE) para preparar las aportaciones españolas ante los congresos internacionales que la UGI celebra cada cuatro años (tabla 1). Además, la Real Sociedad Geográfica edita anualmente el Boletín de la Real Sociedad Geográfica, la publicación decana de geografía en España, que incluye habitualmente trabajos de investigación en didáctica de la geografía.

Si se trasciende el mero ámbito de la geografía y se contempla la investigación en didáctica de las ciencias sociales, la Asociación Universitaria de Profesorado en Didáctica de las Ciencias Sociales (AUPDCS) organiza anualmente los simposios internacionales de didáctica de las ciencias sociales. A pesar de tener un nombre similar, en el ámbito latinoamericano, estos han sido organizados por el grupo DHIGECS de la Universidad de Barcelona y, recientemente, por la red de investigación Red 14, que también promueve los seminarios de investigación en enseñanza de las ciencias sociales. El grupo GREDICS de la Universidad Autónoma de Barcelona organiza anualmente las jornadas internacionales de investigación en didáctica de las ciencias sociales. En todos y cada uno de estos eventos científicos, a pesar de la habitual preeminencia de 
intervenciones relacionadas con la didáctica de la historia, se han presentado resultados de investigaciones relacionadas con la educación geográfica, como hemos podido comprobar en primera persona y como puede constatarse en las actas de los mismos, especialmente en las ediciones de los simposios de la AUPDCS.

En relación con las impresiones en formato de revista, hay dos opciones en España para publicar los trabajos de investigación en didáctica de la geografía. Primero, las revistas de didáctica de las ciencias sociales como Iber: Didáctica de las Ciencias Sociales, Enseñanza de las Ciencias Sociales, Didáctica de las Ciencias Experimentales y Sociales y Con-Ciencia Social, así como otras generalistas de ciencias de la educación y pedagogía. Segundo, las revistas de geografía, comenzando por las dos incluidas en JCR, el Boletín de la Asociación de Geógrafos Españoles y Scripta Nova: Revista Electrónica de Geografía y Ciencias Sociales. La propia plataforma de Geocrítica alberga otras dos revistas digitales que también incluyen regularmente, sobre todo la segunda, trabajos de investigación en didáctica: Biblio3W: Revista Bibliográfica de Geografia y Ciencias Sociales y Ar@cne: Revista Electrónica de Recursos de Internet sobre Geografía y Ciencias Sociales. Otras revistas, Documents d'Anàlisi Geogràfica, han dedicado números monográficos a la enseñanza de la geografía (vol. 56 (2)), además del presente. Estudios Geográficos y Anales de Geografía de la Universidad Complutense son, entre otras, revistas que han publicado de forma poco habitual artículos de didáctica de la geografía.

En Cataluña, Treballs de la Societat Catalana de Geografia ha recogido algún trabajo esporádico de educación geográfica. No obstante, la página web de esta asociación científica (<http://ensenyament-geografia.espais.iec.cat $>$ ) ofrece una gran cantidad y calidad de recursos didácticos para la enseñanza del espacio geográfico.

\section{Conclusiones}

La primera conclusión que obtenemos de este análisis por la reciente producción científica en didáctica de la geografía es el incremento de la cantidad y de la diversidad de proyectos y publicaciones, libros, revistas científicas y recursos didácticos, como expresión del creciente interés por los temas educativos por parte de la comunidad de geógrafos. La dificultad que supone entender las complejas relaciones espaciales del mundo global actual y la revolución que han supuesto las tecnologías de la información geográfica o los nuevos paradigmas del aprendizaje —inteligencias múltiples, neuroeducación — han significado una revisión de los planteamientos establecidos sobre qué geografía enseñar y cómo enseñar geografía. La geografía se ha convertido así en una asignatura de creciente importancia en los sistemas educativos, por ofrecer conocimientos que sirven para descodificar las estructuras sociales, para desarrollar el pensamiento espacial y para contribuir a la adquisición de competencias ciudadanas.

A pesar de lo anterior, la geografía no siempre se ha visto reconocida en los currículos escolares, con más horas de clase de geografía por curso, o con 
presencia obligatoria en todos los cursos de la enseñanza primaria y secundaria. Ello se debe, en parte, a que se sigue percibiendo la geografía como una disciplina académica que reproduce los conocimientos de la ciencia referente. Sin embargo, la investigación educativa ha demostrado que los contenidos geográficos han tenido mayor presencia en la docencia en el aula cuando se integran en asignaturas más generales - de ciencias sociales o social studies- o cuando se vinculan a asignaturas STEM para desarrollar habilidades tecnológicas con los sistemas de información geográfica. El ejemplo del geospatial semester es una buena prueba de ello. En definitiva, se ha producido una evidente disociación entre reconocimiento escolar de la geografía y consolidación del conocimiento científico geográfico-educativo.

No obstante, este incremento constante de producción científica en didáctica de la geografía ha trascendido el ámbito universitario y, debido a su propia naturaleza de conocimiento teórico-práctico, ha alcanzado una gran difusión entre el profesorado de educación primaria y especialmente entre el de secundaria. Las tres referencias citadas al inicio de 1965, 1982 y 1992 ya forman parte del subconsciente colectivo docente. Y la nueva Declaración de 2016 ya ha sido traducida al chino y al español, para llegar de forma más directa a muchos más profesores. $\mathrm{Al}$ menos tantos como son conocedores de las actividades de la propia Comisión de Educación Geográfica en referencia a dos iniciativas: una de mayor tradición y participación internacional, como es la Olimpiada Geográfica (iGEO), que se reproduce a escala nacional o local en muchos ámbitos, y otra, más puntual, como ha sido el reciente concurso de storymaps del Año Internacional para la Comprensión Global. Otros cientos de profesores de geografía en educación escolar participan año tras año en los tres eventos científicos citados, siempre abiertos a recibir comunicaciones y experiencias procedentes de estas "fuentes primarias» del conocimiento educativo geográfico: los congresos del National Council for Geographic Education, de la Geographical Asociation o del Grupo de Didáctica de la AGE.

Una cuarta conclusión reside en la indiscutible disimetría territorial en la producción de la didáctica de la geografía. El ámbito anglosajón (Estados Unidos, Reino Unido y Australia) produce la mayor parte de las publicaciones, en inglés, que no siempre son traducidas al español. Otros ámbitos geográficos como el sudeste asiático o el resto de países europeos participan en esta hegemonía de publicar en inglés. Sin embargo, las iniciativas descritas en España e Iberoamérica han situado a la lengua española como segunda productora mundial de trabajos científicos en didáctica geográfica.

Finalmente, en una lengua o en otra, resulta irrefutable la importancia de las sociedades geográficas - nacionales e internacionales_- así como las redes de profesorado de geografía. Sin ellas, sin sus congresos, sin sus actividades, sin sus debates internos, buena parte de la producción descrita no se habría producido. En resumen, el futuro de la geografía escolar, de la didáctica de la geografía, de la investigación educativa en geografía depende en buena medida del dinamismo de las sociedades geográficas analizadas. Por eso, su frenética 
actividad nos permite afrontar con una cierta tranquilidad los retos que tiene ante sí la educación geográfica.

\section{Referencias bibliográficas}

BAILeY, A. (1981). «La didáctica de la geografía: Diez años de evolución». Geocrítica: Cuadernos Críticos de Geografía Humana, 36, 1-26.

Baker, T.; Battersby, S.; Bednarz, S.; Bozin, A.; Kolvoord, B.; Moore, S.; SinTON, D. y UTTAL, D. (2015). «A Research Agenda for Geospatial Technologies and Learning». Journal of Geography [en línea], 114, 3. <https://doi.org/10.1080/00221341.2014.950684>

BEDNARZ, S. (2004). "Geographic information systems: A tool to support geography and environmental education?». Geojournal [en línea], 60, 191-199. <https://doi.org/10.1023/B:GEJO.0000033574.44345.c9>

Bednarz, S.; Hefrron, S. y Huynh, N. (eds.) (2013). A road map for 21st century geography education. Washington, DC: Association of American Geographers.

Brooks, C.; ButT, G. y FArgher, M. (eds.) (2017). The Power of Geographical Thinking [en línea]. Springer. $<$ https://doi.org/10.1007/978-3-319-49986-4>

ButT, G. (2015). MasterClass in Geography Education. Londres: Bloomsbury.

CAPEL, H.; Luis, A. y URTEAGA, L. (1984). La geografía ante la reforma educativa: Geocrítica. Cuadernos Críticos de Geografía Humana, 53, 1-79.

Castellar, S.; Souza, L. de y CopetTi, H. (2012). Didática da Geografía: Aportes teóricos e metodológicos. São Paulo: Xama Editora / Universidad de São Paulo.

Catling, S. y Willy, T. (2009). Teaching Primary Geography. Exeter: Learning Matters.

Comes, P. (1998). «El espacio en la didáctica de las Ciencias Sociales». En: TREPAT, C. y Comes, P. El tiempo y el espacio en la didáctica de las Ciencias Sociales. Barcelona: Graó, 123-190.

CONWAY, K. y ARAYA, F. (2011). «Discussing the Geography of Sustainable Development through an International Online Collaboration with Students in Chile and the USA». Journal of Geography in Higher Education, 35 (2), 265-279.

Demirci, A.; Miguel, R. de y BednarZ, S. (eds.) (2017). Geography Education for Global Understanding. Springer.

DONERT, K. (ed.) (2010). Using GeoInformation in European Geography Education. Roma: IGU / Home of Geography.

GERBER, R. (ed.) (2003). International handbook on geographical education [en línea]. Dordrecht: Kluwer Academic Publishers. <https://doi.org/10.1007/978-94-017-1942-1>.

Gerber R. y Williams, M. (2002). "An overview of research in geographical education». En: Smith, M. Aspects of Teaching Secondary Geography: Perspectives on practice [en línea]. Londres: Routledge, 303-312. <https://doi.org/10.4324/9780203166116>

GersmeHL, P. (2008). Teaching Geography. Londres: Guildford Press.

Graves, N. (1982). New Unesco Source Book for Geography Teaching. Londres: Longman / UNESCO. Edición española: Nuevo método para la enseñanza de la geografía. Barcelona: Teide; París: UNESCO, 1989.

HAUbRICH, H. (ed.) (1982). International Focus on Geographical Education. Braunsweig: Westermann. 
HefFron, S. y Downs, R. (eds.) (2012). Geography for life: National geography standards. Washington, DC: National Council for Geographic Education.

HiggitT, D.; Donert, K.; Healey, M.; Klein, P.; Solem, M. y VajoczKi, S. (2008). «Developing and Enhancing International Collaborative Learning». Journal of Geography in Higher Education [en línea], 32 (1), 121-133. <https://doi.org/10.1080/03098260701731629>

Hill, A. (ed.) (1992). International Perspectives on Geographic Education. Boulder, CO: Center for Geographic Education. Department of Geography.

Kent, W.; Rawling, E. y Robinson, A. (eds.) (2004). Geographical Education: Making Sense of a Shrinking World. Glasgow: IGU-CGE British Sub-Committee.

- (2008). "The role of GIS in digital earth education». International Journal of Digital Earth, 1 (1), 326-346.

KERSKI, J. (2016). Interpreting Our World: 100 Discoveries That Revolutionized Geography [en línea]. Santa Barbara, CA: ABC-CLIO. <https://doi.org/10.1080/00221341.2017.1316300>

Kulke, E.; Lentz, S. y Wardenga, U. (2004). "Geography in Germany». Belgeo: Revue Belge de Géographie [en línea], 1, 81-95. $<$ https://doi.org/10.4000/belgeo.10067>

Jekel, T.; Car., A.; Strobl, J. y Griesebner, G. (2012). GI_Forum 2012: Geovisualization, Society and Learning. Berlín: Wichmann.

JEREZ, O. y CÓRDOBA, M. (2015). «La producción científica sobre didáctica de la geografía en España y su difusión en el siglo XX». En: SEBASTia, R. y TONDA, E. (eds.). Investigar para innovar en la enseñanza de la Geografía. Alicante: Grupo de Didáctica de la Geografía de la Asociación de Geógrafos Españoles, 759-780.

Lambert, D. y Morgan, J. (2010). Teaching geography 11-18: A conceptual approach. Maidenhead: Open University Press.

LAMbert, D. y JONes., M. (eds.) (2013). Debates in geography education. Londres: Routledge.

LÁZARO, M.L. de (2013). «EUROGEO: Una asociación europea enfocada a la investigación y a impulsar una docencia innovadora, que hoy marca el futuro de la Geografía en Europa». Estudios Geográficos [en línea], LXXIV (275), 715-727. <https://doi.org/10.3989/estgeogr.201326>

LÁZaro, M.L. de; Miguel, M.L. de y GonZÁlez, M.J. (2014). «Real Sociedad Geográfica: An european digital-earth excellence centre: a network for informal learning processes». En: Iceri, 2014 Abstracts. Sevilla: IATED Academy, 1024-1025.

lázaro, M.L. de; Sebastiá, R.; Tonda, E.; Marrón, M.J. y GonZÁlez, J.L. (2013). "Grupo de Didáctica de la Geografía». En: Lasanta, T. y Martin, J. (eds.). La investigación geográfica en España (1990-2012). Madrid: AGE-IPE.

Lidstone J. y Williams, M. (eds.) (2006). Geographical Education in a Changing World: Past Experience, Current Trends and Future Challenges. Dordrecht: Springer.

Lorda, M. y Prieto, M. (2016) (eds.). Didáctica de la Geografía: Debates comprometidos con la actualidad: enseñanza e investigación en la formación docente. Bahía Blanca: Edi-UNS.

Marrón, M.J. y Moreno, A. (1995). Enseñar geografía: De la teoría a la práctica. Madrid: Síntesis.

MarsDen, W. (ed.) (1984). Teacher Education Models in Geography: An International Comparison. Kalamazoo: Western Michigan University Press.

Mével, Y. y Tutiaux-Guillon, N. (2013). Didactique et enseignement de l'HistoireGéographie au Collège et au Lycée. París: Éditions Publibook Université. 
Miguel, R. de (2016). «Pensamiento espacial y conocimiento geográfico en los nuevos estilos de aprendizaje». En: IGLESIAS, R. et al. (eds.). Nativos digitales y geografía en el siglo XXI: Educación geográfica y estilos de aprendizaje. Sevilla: Asociación de Geógrafos Españoles / Universidad Pablo de Olavide de Alicante, 11-39.

Miguel, R. de; Claudino, S. y Souto, X.M. (2016a). "La utopía de la educación geográfica en las declaraciones internacionales de la UGI». En: BENACH, N. et al. (eds). Las utopías y la construcción de la sociedad del futuro. Barcelona: Universidad de Barcelona.

Miguel, R. de y DonerT, K. (eds.) (2014). Innovative learning Geography in Europe: New challenges for the 21st Century. Newcastle: Cambridge Scholar Publishing.

Miguel, R. de y LÁZARO, M.L. de (2016). «Educating geographers in Spain: Geography teaching renewal by implementing the European Higher Education Area». Journal of Geography in Higher Education, 40 (2), 267-283.

Miguel, R. de; Lázaro, M.L. de; Velilla, J.; Buzo, I. y Guallart, C. (2016b). "Atlas Digital Escolar: Internet, geografía y educación».Ar@cne: Revista Electrónica de Recursos de Internet sobre Geografia y Ciencias Sociales [en línea], 212. <http:// www.ub.edu/geocrit/aracne.htm>.

Miguel, R. de y ScheE, J. van der (2015). «Declaración Internacional sobre Investigación en Educación Geográfica». Didáctica Geográfica, 15, 195-200.

Milson, A.; DeMIRCI, A. y KERSKI, J. (eds.) (2012). International Perspectives on Teaching and Learning with GIS in Secondary Schools [en línea]. Dordrecht: Springer. <https://doi.org/10.1007/978-94-007-2120-3>

Muñiz, O.; Demirci, A. y Van Der Schee, J. (eds.) (2015). Geospatial Technologies and Geography Education in a Changing World. Tokyo: Springer.

MuÑIz, O.; Solem, M. y BoeHM, R. (eds.) (2016). Learning progressions in Geography Education. Springer.

NATIONAL RESEARCH COUNCIL (2006). Learning to think spatially: GIS as a support system in the K-12 curriculum. Washington, DC: The National Academies Press.

Purnell, K.; Lidstone, J. y Hodgson, S. (eds.) (2006). Changes in Geographical Education: Past, Present, and Future. Proceedings of the IGU-CGE Symposium. Brisbane: Royal Geographical Society of Queensland.

ReINFried, S.; SCHLEICHER, Y. y ReMPFLER, A. (eds.) (2007). Geographical Views on Education for Sustainable Development. Proceedings of the Lucerne-Symposium. Lucerne: IGU-CGE.

RoBerts, M. (2013). Geography through Enquiry: Approaches to teaching and learning in the secondary school. Sheffield: Geographical Association.

Robic, M. y Rosemberg, M. (eds.) (2016). Géographier aujourd'hui: Enseigner la géographie au collège et au lycée. París: Adpat Editions.

SchmeInCK, D. (ed.) (2009). Teaching in and for Europe. Berlín: Mech und Buch.

SEBASTIÁ, R. y TONDA, E. (2011). «Características y evolución de la revista didáctica geográfica». Didáctica Geográfica, 12, 19-48.

- (2012). «Diez años de didáctica de la geografía a través de los congresos nacionales del Grupo de Didáctica de la Geografía (2000-2010)». En: Miguel, R. de; LAZARO, M.L. de y MARRÓN, M.J. (eds.). La educación geográfica digital. Zaragoza: Asociación de Geógrafos Españoles. Grupo de Didáctica de la Geografía, 59-72.

- (2015). «Difusión de la investigación e innovación en la revista Didáctica Geográfica». En: SEBASTIA, R. y TONDA, E. (eds.). Investigar para innovar en la enseñanza de la geografía. Alicante: Grupo de Didáctica de la Geografía de la Asociación de Geógrafos Españoles, 817-828. 
- (eds.) (2017). Enseñanza y aprendizaje de la geografía para el siglo XXI. Alicante: Universidad de Alicante.

Souto, X. (1998). Didáctica de la geografía: Problemas sociales y conocimiento del medio. Barcelona: Serbal.

SouTO, X. y FiTA, S. (2014). «Las diferencias sociales y la cultura escolar: El Geoforo Iberoamericano en 2014». Biblio 3W: Revista Bibliográfica de Geografía y Ciencias Sociales [en línea], XIX, 1105. <http://www.ub.edu/geocrit/bw-ig.htm>.

STANDish, A. (2012). The False Promise of Global Learning: Why Education Needs Boundaries. Londres: Continuum.

Stoltman, J. (1976). International Research in Geographical Education. Kalamazoo: Western Michigan University Department of Geography.

Stoltman, J. y LiDSTONE, J. (2017). «The End of the Beginning: The emerging role of International Research in Geographical and Environmental Education (IRGEE)». International Research in Geographical and Environmental Education [en línea], 26 (I), 87-90. <https://doi.org/10.1080/10382046.2017.1295580>

TOnda, E. y SEbastia, R. (2012). «Diez años de didáctica de la geografía a través de los congresos nacionales del Grupo de Didáctica de la Geografía (1988-1998)». En: Miguel, R. de; LÁZARO, M.L. de y MARRÓN, M.J. (eds.). La educación geográfica digital. Zaragoza: Asociación de Geógrafos Españoles. Grupo de Didáctica de la Geografía, 93-106.

Tovar, S.; Ruiz, M. y Plans, P. (1980). «Bibliografía española de Didáctica Geográfica». Didáctica Geográfica, 5, 27-74.

UNESCO (1965). Unesco Source Book for Geography Teaching. Londres: LongmanUNESCO. Edición española: Método para la enseñanza de la geografía. Barcelona / París: Teide / UNESCO, 1966.

Whewell, C.; Brooks, C.; Butt, G y Thurston, A. (2011). Curriculum making in Geography. Londres: Institute of Education / IGU-CGE.

ZadroZnY, J.; Mcclure, C.; LeE, J. y Jo, I. (2016). «Designs, Techniques, and Reporting Strategies in Geography Education: A Review of Research Methods». Review of International Geographical Education Online, 6 (3), 216-233. 